\title{
Cintilografia Miocárdica para Estudo da Inervação Simpática
}

As inervações simpática e parassimpática do coração regulam a função miocárdica, freqüência cardíaca e fluxo sangüíneo miocárdico em indivíduos normais e portadores de doença cardíaca. Entre essas, se destacam as síndromes isquêmicas, insuficiência cardíaca, morte súbita e diabetes. A meta-iodo-benzil-guanidina (MIBG) é um análogo da guanetidina, um falso neurotransmissor, que é captado pelos neurônios adrenégicos à semelhança da norepinefrina (figura 1 ), mas que não participa do metabolismo intracelular. as características descritas, a MIBG vem sendo utilizada na avaliação in vivo do papel do sistema nervoso simpático cardíaco em condições fisiológicas e fisiopatológicas. Tais imagens representam a densidade dos receptores e o tônus simpático.

A MIBG pode ser marcada com iodo-123 ou iodo131. Quando marcada com iodo-131 (radioisótopo beta e gama emissor de média energia, meia-vida física de 8 dias), a dose administrada deve ser baixa para não expor 0 paciente à dosimetria alta.

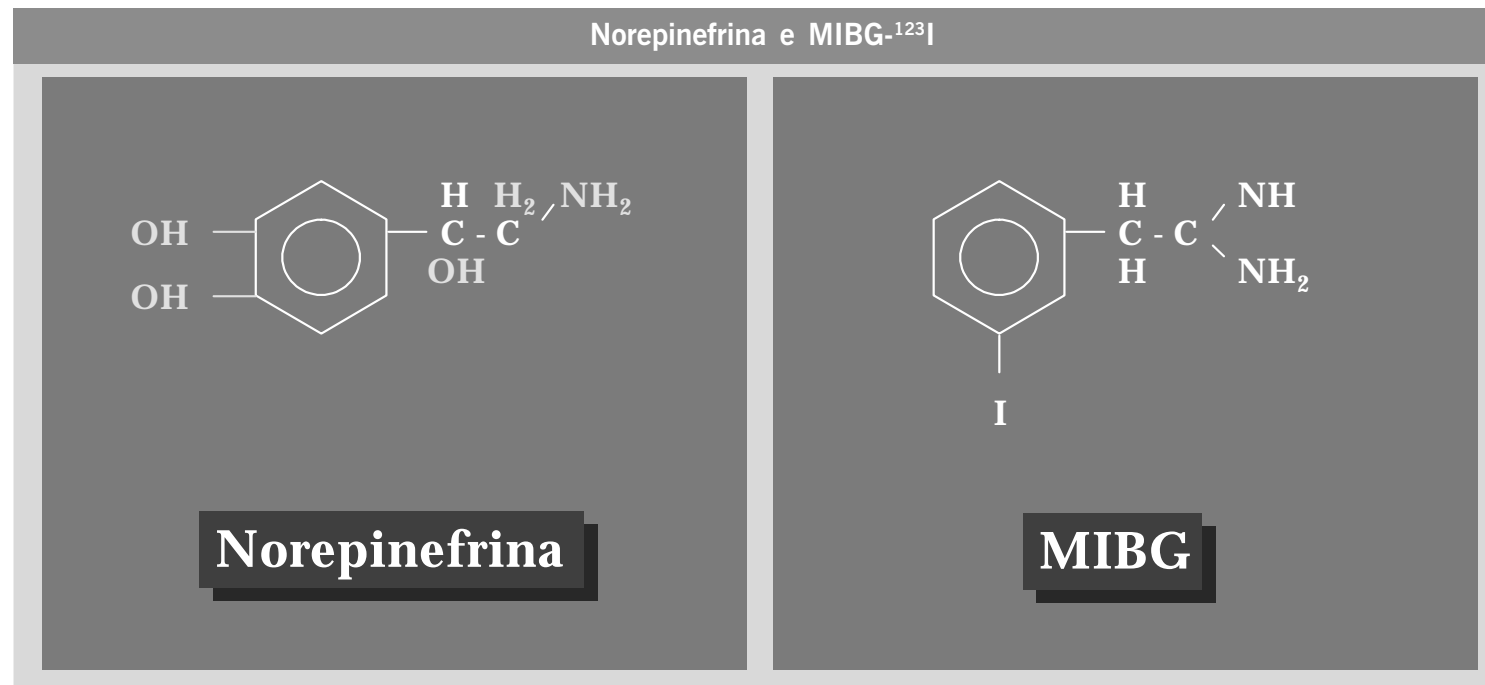

Figura 1 - Mostra a fórmula da norepinefrina e da meta-iodo-benzilguanidina, análogo que permite a marcação "in vivo" da inervação autonômica do ventrículo esquerdo.

A sua localização no músculo cardíaco é dependente dos mecanismos de recaptação da norepinefrina, com entrada do agente nas vesículas de estocagem nas terminações nervosas pré-sinápticas e nas células da medula adrenal. 0 que reforça essa teoria é a redução da captação quando se administra previamente a reserpina, desmetilimipramina ou antidepressivos tricíclicos. Bloqueadores alfa e beta não interferem com sua captação.

A captação da MIBG pelo músculo cardíaco se faz por um mecanismo neuronal de alta afinidade (uptake-1 ou reuptake) e não neuronal de baixa atividade (uptake-2). O fato de o efluxo da MIBG do compartimento não neuronal ser rápido em relação ao neuronal, facilita a avaliação da distribuição autonômica cardíaca. A MIBG não é metabolizada pela monoamino-oxidase (MAO) nem pela catecol-orto-metil-transferase (COMT). Sua captação é maior nas áreas de inervação normal e menor nas áreas
O paciente deve receber iodo frio, na forma de iodeto, para bloquear a tireóide. As imagens planas podem ser semiquantificadas, usando pulmão ou mediastino como referência. Quando marcada com iodo-123 (emissor gama puro de baixa energia, meia-vida física de 13 horas), as doses podem ser maiores. Bloqueia-se também a tireóide, e as cintilografias são obtidas na forma plana ou tomográfica (figura 2) com o objetivo de ver a biodistribuição cardíaca e, se necessário, também quantificar de forma relativa.

Técnica - Para a realização de imagens com a MIBG, é necessário que o paciente se submeta a um preparo prévio, que consiste em suspensão dos fármacos que interferem com a captação: reserpina e guanetidina (depleção de grânulos); fenilefrina, pseudo-efedrina, fenilpropanolamina (agonistas); antipsicóticos; antidepressivos tricíclicos; bloqueadores dos canais de cálcio (exceto di-hidropiridínicos); e betabloqueadores. É necessário também, o bloqueio para proteção da 


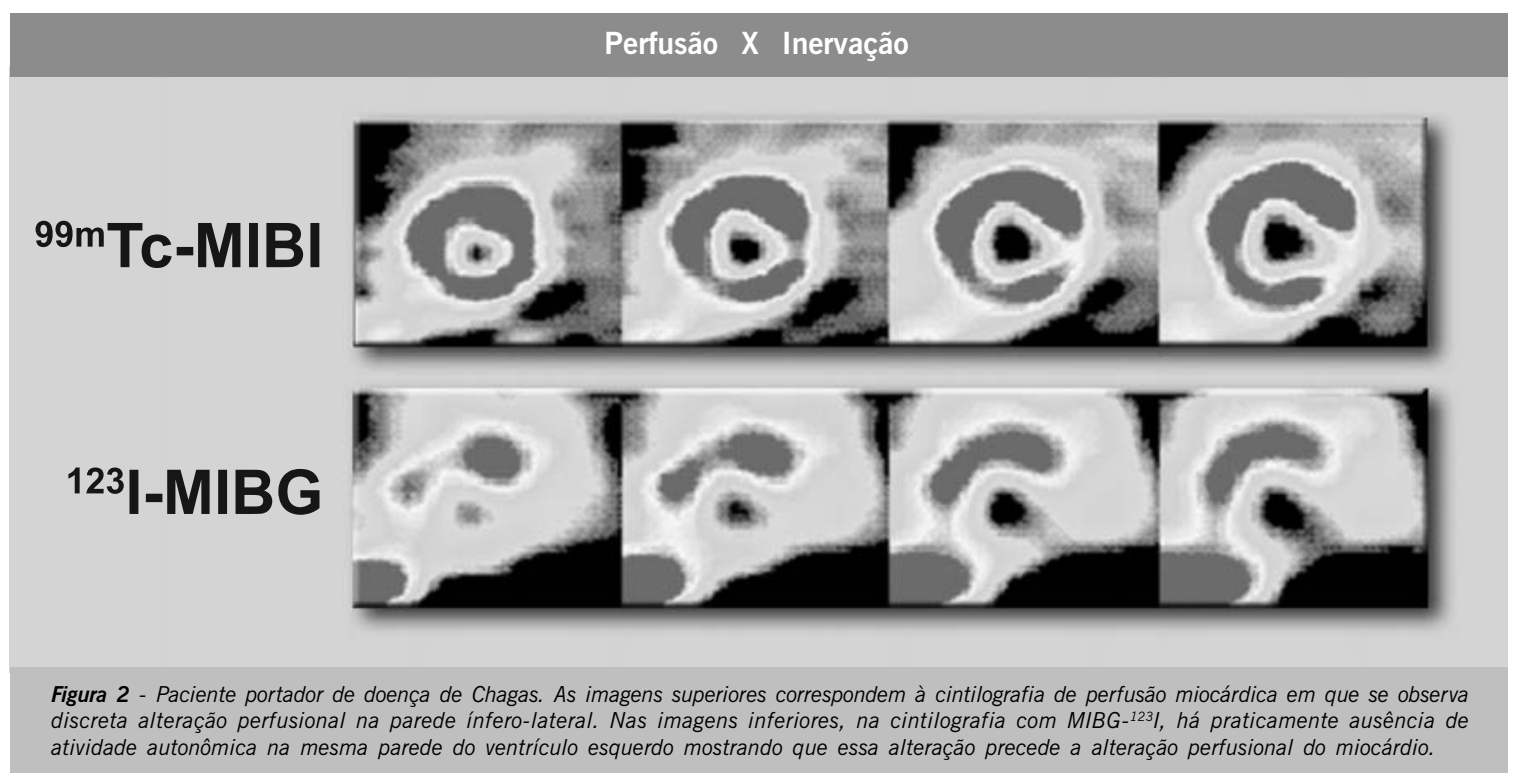

tireóide com iodeto ou lugol, pois a MIBG é marcada com iodo radioativo, sendo mais freqüente com o iodo123. As imagens planas podem ser realizadas utilizandose uma dose de 37 a $100 \mathrm{MBq}(1 \mathrm{mCi}=37 \mathrm{MBq})$ de MIBG marcada com iodo-123 ou iodo-131. Adquiremse pelo menos 1.000 .000 de contagens nas projeções anterior e oblíqua anterior esquerda a $45^{\circ}$ do tórax, 20 minutos (imagens precoces) e 2-4 horas (imagens tardias) após a administração do radiofármaco. Pode-se calcular a relação coração-mediastino (normal: menor que 1,8 ) e a taxa de clareamento (normal: menor que 10\%). As imagens tomográficas também podem ser adquiridas nos tempos precoce e tardio. Faz-se a reconstrução das imagens em 3 planos: eixo curto, eixo longo horizontal e longo vertical. Verifica-se a taxa de clareamento (por parede miocárdica), os mapas polares (o que permite comparação com grupo normal), ou pode-se comparar a intensidade de contagens nas paredes do ventrículo esquerdo com a sua cavidade.

\section{Aplicações Clínicas}

Doença cardíaca isquêmica - As fibras simpáticas estão distribuídas da base até o ápice do coração e penetram do epicárdio para o endocárdio paralelo aos vasos coronarianos. As fibras parassimpáticas são em muito menor número e penetram do endocárdio para a superfície do epicárdio. Quando há infarto sem onda $Q$, a denervação corresponde à zona do infarto, enquanto que, nos eventos com onda $\mathrm{Q}$, a área denervada é maior que a do infarto. $\mathrm{A}$ denervação ocorre por necrose das fibras, mas essas também se alteram temporariamente pela isquemia. $\mathrm{Na}$ isquemia miocárdica com recuperação da perfusão, podese verificar denervação transitória. Isso demonstra que os miócitos são menos sensíveis à isquemia do que as fibras nervosas. Outro fato importante é que a área denervada é hipersensível a catecolaminas, sendo uma das justificativas do aumento de arritmias nos eventos agudos.
Diabete mellitus - Quando há diabete mellitus com neuropatia autonômica, aumentam as taxas de morbidade e mortalidade. Os principais efeitos são hipotensão postural, taquicardia de repouso, alteração na regulação do fluxo sangüíneo miocárdico, disfunção ventricular e isquemia silenciosa. Pacientes com neuropatia autonômica diabética apresentam maiores alterações na cintilografia com MIBG mesmo na ausência de arteriosclerose coronariana. Por outro lado, melhora no controle da glicemia resulta em restauração da inervação simpática observada por imagens com MIBG. Isquemia silenciosa é mais comum em pacientes diabéticos e está associada com morte súbita. Tem sido postulado que pacientes com diabetes sofrem interrupção dos sensores aferentes e eferentes das fibras cardíacas nervosas, o que pode levar à perda da percepção da dor. Não foram demonstradas diferenças entre cintilografia de perfusão miocárdica e de MIBG em pacientes com diabetes com ou sem isquemia silenciosa.

Insuficiência cardíaca congestiva e cardiomiopatia Pacientes com insuficiência cardíaca congestiva (ICC) têm evidências de aumento da ativação do sistema nervoso simpático manifestado pelo elevado nível de norepinefrina circulante, com sinais de alteração da função cardíaca simpática. Uma das formas de medir a função nervosa simpática do coração é avaliar a capacidade dos nervos terminais em captar catecolaminas pelo transportador uptake-1. Essa avaliação pode ser feita de forma não invasiva com MIBG medindo a relação da captação cardíaca com a captação do mediastino (C/M). Essa relação declina com a progressão da ICC. Por isso, quanto maior a redução, pior o prognóstico. Além disso, essas anormalidades se correlacionam com a FEVE, classe funcional (NYHA) e alterações histopatológicas. A cintilografia cardíaca com MIBG na ICC mostra alterações difusas, diferentemente da doença isquêmica. $\mathrm{O}$ grau de anormalidade da relação $\mathrm{C} / \mathrm{M}$ mostrou ser preditor independente de mortalidade e melhor do que a fração de ejeção ventricular esquerda, volumes 
ventriculares e da classe funcional.

$\mathrm{Na}$ doença de Chagas, há alteração da inervação simpática que pode ser observada pela cintilografia com MIBG. Partes de pacientes na forma indeterminada mostram hipocaptação inferior e apical. Quando estudados simultaneamente com a perfusão miocárdica, foram observados defeitos de inervação maiores que os de perfusão, sugerindo que a denervação precede a fibrose nessa doença, como mostra a figura 2 .

A terapia de ICC com betabloqueadores melhora as taxas de morbidade e mortalidade. Durante a terapia, pode ser observada, pela cintilografia com MIBG, a melhora progressiva do índice $\mathrm{C} / \mathrm{M}$. A cintilografia com MIBG mostra melhora na concentração do marcador antes mesmo da resposta da FEVE, com valor prognóstico adicional daqueles que irão responder ao tratamento. $\mathrm{Na}$ ICC causada por ação tóxica de quimioterápicos como a família da antraciclina, a alteração da função adrenérgica medida pela MIBG precede a queda da FEVE, mostrando ser instrumento sensível para monitorar cardiotoxidade.

Transplante Cardíaco- Todos os pacientes submetidos a transplante cardíaco apresentam denervação miocárdica que persiste por tempo variável após a cirurgia, habitualmente até 12 meses, podendo ser demonstrada pela MIBG. O processo de reinervação parece começar na parede ântero-lateral do VE. Nessa fase, alguns pacientes voltam a apresentar angina e modificações na variabilidade de freqüência cardíaca. Outro marcador de inervação, a 11-hidroxi-efedrina, também mostra maior captação da região irrigada pela artéria descendente anterior. Esses estudos confirmam o importante papel na integridade da regulação do fluxo sangüíneo miocárdico.

\section{Conclusões}

A análise do sistema nervoso simpático com MIBG parece ser útil na avaliação da ICC, da morte súbita, arritmias, cardiopatia isquêmica, diabetes mellitus e no transplante cardíaco. Também pode ser importante na avaliação de resultados terapêuticos. Este método tem sido utilizado, predominantemente, em pequenos estudos e em pesquisas clínicas, com resultados promissores, particularmente em pacientes com ICC. No entanto, são necessários mais dados obtidos através de estudos multicêntricos randomizados, para a confirmação do valor diagnóstico e prognóstico da cintilografia com MIBG para a avaliação do sistema nervoso autonômico cardíaco.

\section{REFERÊNCIAS}

1. Sisson JC, Shapiro B, Meyers L et al. Metaiodobenzylguanidine to map scintigraphically the adrenergic nervous system in man. J Nucl Med 1987; 28:1625-36.

2. Kline RC, Swanson DP, Wieland DM et al. Myocardial imaging in man with I-123 meta-iodobenzyilguanidine. J Nucl Med 1981; 22:129-32.

3. Patel A, Iskandrian AE. MIBG imaging. J Nucl Med 2002;1:75-94.

4. Langer A, Freeman MR, Josse RG et al. Metaiodobenzylguanidine imaging in diabetes mellitus: assessment of cardiac sympathetic denervation and its relation to autonomic dysfunction and silent myocardial ischemia. J Am Coll Cardiol 1995; 25:610-8.
5. Zipes DP. Influence of myocardial ischemia and infarction on autonomic innervation of heart. Circulation 1998; 78:1008-19.

6. Lotze $U$, Kober A, Kaepplinger $S$ et al. Cardiac sympathetic activity as measured by myocardial 123-I-metaiodobenzyilguanidine uptake and heart rate variability in idiophatic dilated cardiomyopathy. Am J Cardiol 1999; 83:1548-57.

7. Giorgi MCP, Hironaka FH, lanni B et al. Myocardial perfusion and sympathetic system in Chagas' heart disease: preliminary data. J Nucl Cardiol 1995; 2: S25. 